\title{
Utility of ultrasound-guided transversus abdominis plane block for day-case inguinal hernia repair
}

\author{
Luciano Frassanito ${ }^{1}$, Sara Pitoni ${ }^{1}$, Gianluigi Gonnella ${ }^{1}$, Sergio Alfieri ${ }^{2}$, \\ Miryam Del Vicario ${ }^{1}$, Stefano Catarci ${ }^{1}$, and Gaetano Draisci ${ }^{1}$ \\ ${ }^{1}$ Institute of Anesthesiology and Intensive Care Medicine, ${ }^{2}$ Institute of Digestive Surgery, Catholic University of the \\ Sacred Heart, "A. Gemelli" University Polyclinic Foundation, Rome, Italy
}

\begin{abstract}
Background: The transversus abdominis plane (TAP) block is a regional anesthesia technique that effectively reduces the pain intensity and use of analgesia in abdominal surgery. The aim of this study was to determine the utility of the ultrasound-guided TAP block in improving the efficacy of the ultrasound-guided ilioinguinal/iliohypogastric nerve (IIN/ IHN) block for intraoperative anesthesia and postoperative pain control in day-case inguinal hernia repair (IHR).

Methods: We conducted a descriptive study of patients undergoing elective primary unilateral open IHR. Fifty-nine patients were divided into two groups according to the anesthetic technique used: ultrasound-guided TAP block plus ultrasound-guided IIN/IHN block (TAP group) vs. ultrasound-guided IIN/IHN block alone (IIN/IHN group). The outcome measures were the adequacy of anesthesia during surgery and postoperative analgesia.

Results: Four patients (12.5\%) in the TAP group and 10 patients (37.0\%) in the IIN/IHN group experienced inadequate anesthesia and needed systemic sedation $(\mathrm{P}<0.05)$. No significant differences in additional local anesthetic volume were found between the two groups. Patients in the TAP group reported lower pain scores at the end of surgery $(0.4 \pm 0.8$ vs. $2.1 \pm 2.5, \mathrm{P}<0.01)$, at 2 hours after surgery $(0.8 \pm 1.3$ vs. $3.0 \pm 2.2, \mathrm{P}<0.01)$, at discharge $(1.4 \pm 1.2$ vs. $4.3 \pm 2.2, \mathrm{P}<0.01)$, and at 24 hours $(1.5 \pm 1.1$ vs. $4.5 \pm 2.3, \mathrm{P}<0.01)$.

Conclusions: The combination of the TAP and IIN/IHN blocks is associated with better intraoperative anesthesia and lower postoperative pain scores compared with the IIN/IHN block alone.
\end{abstract}

Key Words: Ilioinguinal nerve, Inguinal hernia, Nerve block, Postoperative pain, Regional anesthesia, Ultrasound.

Corresponding author: Miryam Del Vicario, M.D.

Institute of Anesthesiology and Intensive Care Medicine, Catholic University of the Sacred Heart, "A. Gemelli" University Polyclinic Foundation, Largo A. Gemelli 8, Rome 00168, Italy

Tel: 39-0630154507, Fax: 39-063013450

Email: miryam.delvicario@gmail.com

ORCID: http://orcid.org/0000-0001-6972-8305

Received: June 3, 2016.

Revised: July 22, 2016 (1st); August 18, 2016 (2nd).

Accepted: August 25, 2016.

Korean J Anesthesiol 2017 February 70(1): 46-51 https://doi.org/10.4097/kjae.2017.70.1.46

\section{Introduction}

Inguinal hernia repair (IHR) is one of the most commonly performed surgical procedures worldwide. Different anesthesia techniques, either alone or in association, can be used to carry out IHR. These include general anesthesia, sedation, spinal anesthesia, ilioinguinal/iliohypogastric nerve (IIN/IHN) block, and infiltration of the surgical field with a long-acting local anesthetic (LA) agent [1-5].

Several retrospective studies and randomized controlled trials have demonstrated the clinical and pharmacoeconomic superiority of local anesthesia over spinal and general anesthesia $[2,4,5]$. Other reports have shown that LA infiltration is as-

(c) This is an open-access article distributed under the terms of the Creative Commons Attribution Non-Commercial License (http://creativecommons.org/ licenses/by-nc/4.0/), which permits unrestricted non-commercial use, distribution, and reproduction in any medium, provided the original work is properly cited. 
sociated with lower postoperative visual analogue scale (VAS) scores, reduced analgesic consumption, and longer times to use of the first rescue analgesic [3,6-9].

The IIN/IHN block is widely used as postoperative analgesic technique in inguinal surgery. It involves blockade of the IIN and IHN terminations anteromedial to the anterior superior iliac spine [10]. Despite its seemingly easy application, a relatively high failure rate of $10-25 \%$ has been reported [11]. A significant increase in the overall success rate of adequate blocks has been described using an ultrasound (US)-guided technique [11-13].

The transversus abdominis plane (TAP) is an intermuscular plane between the internal oblique and transversus abdominis muscles. The TAP block is a regional anesthesia technique that provides analgesia to the parietal peritoneum as well as to the skin and muscles of the anterior abdominal wall from the T7 to L1 dermatomes [14,15]. This technique can be easily performed under US guidance and has been found to be effective in reducing the pain intensity and analgesic consumption after abdominal surgery [15-18]. Despite its wide range of applications, the role of the TAP block in anesthesia for IHR remains understudied. The aim of this descriptive observational study was to determine the utility of the US-guided TAP block in improving the efficacy of the US-guided IIN/IHN block for intraoperative anesthesia and postoperative pain control after day-case inguinal hernia surgery. The primary end point was the adequacy of surgical anesthesia, and the secondary end point was the postoperative pain score.

\section{Materials and Methods}

\section{Study design}

After local ethics committee approval, we conducted a prospective, descriptive, observational study of patients with an American Society of Anesthesiologists physical status of I to III who underwent elective primary unilateral open IHR from August 2013 to January 2015. The patients were divided into two groups according to the anesthetic technique used. The TAP group comprised patients who underwent the US-guided TAP block in addition to the US-guided IIN/IHN block, while the IIN/IHN group comprised patients who underwent the USguided IIN/IHN block alone. The primary outcome was the adequacy of anesthesia (requirements for additional LA infiltration by surgeons and systemic sedation). The secondary outcomes were the postoperative VAS scores at rest and while coughing, measured at different time points during the first 24 hours after surgery (end of surgery and at 120 minutes, discharge, and 24 hours). The exclusion criteria were an inability to provide consent; age $\leq 18$ years; body mass index $\geq 40 \mathrm{~kg} / \mathrm{m}^{2}$; contraindications to regional anesthesia, ketorolac, or paracetamol; chronic hepatic or renal failure; and preoperative treatment with opioids or nonsteroidal anti-inflammatory drugs for chronic pain.

All surgical procedures were performed by the same surgeons according to Lichtenstein's technique (open IHR with mesh). All blocks were performed before the operation by two anesthetists, both experienced in locoregional anesthesia, using a full aseptic technique. During the procedure, standard noninvasive monitoring consisting of a continuous display of electrocardiographic and pulse oximetry parameters and intermittent measurement of noninvasive blood pressure was applied, and supplemental oxygen (2-3 L/min) was administered via a nasal cannula. Midazolam at $0.02 \mathrm{mg} / \mathrm{kg}$ was administered intravenously before the procedure. After administration of locoregional anesthesia, all patients received remifentanil target-controlled infusion (TCI) at $0.75 \mathrm{ng} / \mathrm{ml}$ (pharmacokinetic model by Minto). All patients received $1 \mathrm{~g}$ of paracetamol during surgery. Anesthesia was considered adequate if the patients did not require a further anesthetic procedure with sedation during the intervention. For intraoperative pain, the surgeon performed further infiltration of $5 \mathrm{ml}$ of $0.2 \%$ ropivacaine until a maximum dose of $20 \mathrm{ml}$. For persistent intraoperative pain, deep sedation with propofol TCI at $3 \mu \mathrm{g} / \mathrm{ml}$ (pharmacokinetic model by Schnider) was started, and the patient was ventilated with a facial mask until the end of the surgery. According to our internal protocol, the patients were given instructions on how to use a VAS scale graded from 0 (no pain) to 10 (worst pain imaginable). The VAS scores were recorded at each time point. In the ward, when the VAS score was $>5$, intramuscular ketorolac $(30 \mathrm{mg})$ was administered up to twice a day. For persistent pain, intramuscular tramadol (100 $\mathrm{mg}$ ) was administered. The occurrence of nausea, vomiting, and femoral nerve palsy was evaluated. Patients were discharged home from the outpatient surgery unit on the same day as surgery. The discharge criteria included a VAS score of $\leq 4$ and absence of postoperative nausea and vomiting.

\section{Surgical technique}

All patients underwent operations by the same surgical team. The Ultrapro hernia system (UHS) was used for IHR in both groups. The apex of the UHS mesh was sutured to the pubic tubercle using a 3-0 Vicryl suture, with the lower part in the preperitoneal space under the transverse fascia and the upper part placed to fit the floor of the inguinal canal. The lower border of the mesh was fixed with one stitch to the free edge of the inguinal ligament after creation of an opening to accommodate the spermatic cord. No other stitches were placed.

\section{Ilioinguinal/iliohypogastric nerve block}

In the supine position, after skin disinfection with povidone- 
iodine solution, the abdominal wall was scanned using a $38 \mathrm{~mm}$ broadband linear array transducer probe $(6-12 \mathrm{MHz})$ in the multibeam mode connected to a portable ultrasound unit (Esaote MyLab 30). The probe was covered by a sterile plastic transducer sheath (Vygon, France), and sterile gel (Vygon, France) was applied to the skin. The IIN/IHN block was performed according the technique described by Gofeld and Christakis [11]. The IIN was visualized, usually between the internal oblique and transverse or external oblique muscles and within 1 to $3 \mathrm{~cm}$ from the anterior superior iliac spine. A $70 \mathrm{~mm}, 22 \mathrm{G}$ shortbevel needle (Temena, Germany) was inserted laterally through the entry point of the transducer, and $10 \mathrm{ml}$ of $0.5 \%$ ropivacaine was injected. The solution encircled the nerve and appeared as a distinct hypoechoic structure highlighted by the hyperechoic surrounding fat. Needle placement and spread of the LA were easily seen.

\section{Transversus abdominis plane block}

The medial head of the ultrasound probe was positioned on the anterior wall at the level of the umbilicus, with adjustments made to obtain optimal images until observation of the rectus abdominis. We then moved the probe anterolaterally to obtain a transverse view of the abdominal layers, scanning the abdominal wall between the iliac crest and the subcostal margin. This allowed for visualization of the external oblique, internal oblique, transversus abdominis, and, most deeply, the peritoneal cavity. Using the in-plane technique under real-time US assessment, the needle was inserted from lateral to medial until the injection site was reached (between the aponeurosis of the internal oblique and transversus abdominis muscles). Ropivacaine $0.5 \%$
(20 $\mathrm{ml})$ was injected with intermittent aspiration. The expansion of the LA solution as a hypoechoic shadow between the aponeurosis of the internal oblique and transversus abdominis muscles, pushing the muscle deeper, confirmed the correct placement of the needle.

\section{Statistical analysis}

Statistical analysis was performed using SPSS statistical software (version 20 for Windows; IBM Corp., Armonk, NY, USA). The mean, median, and standard deviation were calculated for all quantitative variables. The normality of the data was checked using the Kolmogorov-Smirnov and Shapiro-Wilk tests of normality. For normally distributed data, means were compared using the t-test for independent samples. For non-normally distributed data, the Mann-Whitney test was applied and interquartile ranges calculated. Qualitative and categorical variables were described as frequencies and proportions. Proportions were compared using the chi-squared test or Fisher's exact test, whichever was applicable. All statistical tests were two-sided and were performed at a significance level of $\mathrm{P}=0.05$.

\section{Results}

Sixty-six patients undergoing surgical IHR with the Lichtenstein technique from August 2013 to January 2015 in the outpatient clinic of our institution were screened. Among them, 59 were studied and 7 were excluded because they did not fulfill the inclusion/exclusion criteria. In particular, five of them did not provide consent, one was allergic to nonsteroidal anti-inflammatory drugs, and one had a body mass index $>40 \mathrm{~kg} / \mathrm{m}^{2}$. Fifty-

Table 1. Baseline Demographic Characteristics

\begin{tabular}{lcccc}
\hline & TAP group $(\mathrm{n}=32)$ & IIN/IHN group $(\mathrm{n}=27)$ & Test & P value \\
\hline Age $(\mathrm{yr})$ & $58 \pm 15$ & $54 \pm 15$ & t-test & NS \\
Male & $29 / 32(90.6)$ & $24 / 27(88.9)$ & Chi-square & NS \\
Right side & $20 / 32(62.5)$ & $14 / 27(51.9)$ & Chi-square & NS \\
\hline
\end{tabular}

Values are expressed as mean \pm SD or number of patients (\%). TAP group: ultrasound-guided TAP block plus ultrasound-guided IIN/IHN block, IIN/ IHN group: ultrasound-guided IIN/IHN block alone.

Table 2. Supplemental Local Anesthetic (LA) Volume Administered by Surgeons, Patients Requiring Sedation with Propofol Target-controlled Infusion, Patients Requiring Rescue Analgesic (Ketorolac), and Unintended Femoral Nerve Block

\begin{tabular}{lcccc}
\hline & IIN/IHN group $(\mathrm{n}=27)$ & TAP group $(\mathrm{n}=32)$ & Test & P value \\
\hline LA volume $(\mathrm{ml})$ & $16.4 \pm 9.8$ & $14.2 \pm 12.1$ & Mann-Whitney U test & NS \\
Inadequate anesthesia & $10 / 27(37)$ & $4 / 32(12.5)$ & Chi-square & $<0.05$ \\
Ketorolac rescue & $16 / 27(59.3)$ & $3 / 32(9.4)$ & Chi-square & $<0.05$ \\
Femoral block & $2 / 27(7.4)$ & $2 / 32(6.3)$ & Chi-square & NS \\
\hline
\end{tabular}

Values are expressed as mean \pm SD or number of patients (\%). TAP group: ultrasound-guided TAP block plus ultrasound-guided IIN/IHN block, IIN/ IHN group: ultrasound-guided IIN/IHN block alone. 
nine patients were therefore evaluated: 32 of them were included in the TAP group and 27 in the IIN/IHN group. The baseline clinical and demographic characteristics at the time of screening were similar between the two groups (Table 1). Intraoperatively, there was no difference in the mean quantity of supplemental LA agents administered by the surgeon because of insufficient analgesia $(16.4 \pm 9.8 \mathrm{ml}$ in the IIN/IHN group vs. $14.2 \pm 12.1 \mathrm{ml}$ in the TAP group, $\mathrm{P} \geq 0.05$ ) (Table 2 ); however, there was a significant difference in the intraoperative pain perception between the two groups. In fact, a greater number of patients required deep sedation with propofol TCI in the IIN/IHN group than in the TAP group (37.0\% vs. $12.5 \%$, respectively; $\mathrm{P}<0.05)$. One of the patients in the IIN/IHN group required general anesthesia.

The postoperative VAS scores at rest were analyzed (Fig. 1). Patients in the TAP group experienced significantly less pain immediately after surgery $(0.4 \pm 0.8$ vs. $2.1 \pm 2.5, \mathrm{P}<0.01)$, at 2 hours $(0.8 \pm 1.3$ vs. $3.0 \pm 2.2, \mathrm{P}<0.01)$, at discharge ( $1.4 \pm 1.2$ vs. $4.3 \pm 2.2, \mathrm{P}<0.01)$, and at 24 hours after surgery $(1.5 \pm 1.1$ vs. 4.5 $\pm 2.3, \mathrm{P}<0.01)$ compared with the IIN/IHN group.

The results of pain on coughing were similar to those of pain at rest: patients in the TAP group reported significantly lower VAS scores on coughing at the end of surgery ( $2.2 \pm 1.8$ vs. 5.3 $\pm 3.2, \mathrm{P}<0.01)$, at 2 hours $(3.1 \pm 2.5$ vs. $6.9 \pm 4.7, \mathrm{P}<0.01)$, at discharge $(2.8 \pm 3.2$ vs. $5.9 \pm 3.3, \mathrm{P}<0.01)$, and at 24 hours after surgery $(2.5 \pm 1.8$ vs. $5.2 \pm 4.7, \mathrm{P}<0.01)$ compared with the IIN/ IHN group.

A higher number of patients in the IIN/IHN group required rescue doses of ketorolac (59.3\% vs. 9.4\%, P < 0.05) (Table 2); two of these patients needed an extra dose of intramuscular tramadol. There was no difference in the incidence of femoral nerve block between the two groups (Table 2). All four cases of femoral nerve palsy resolved spontaneously.

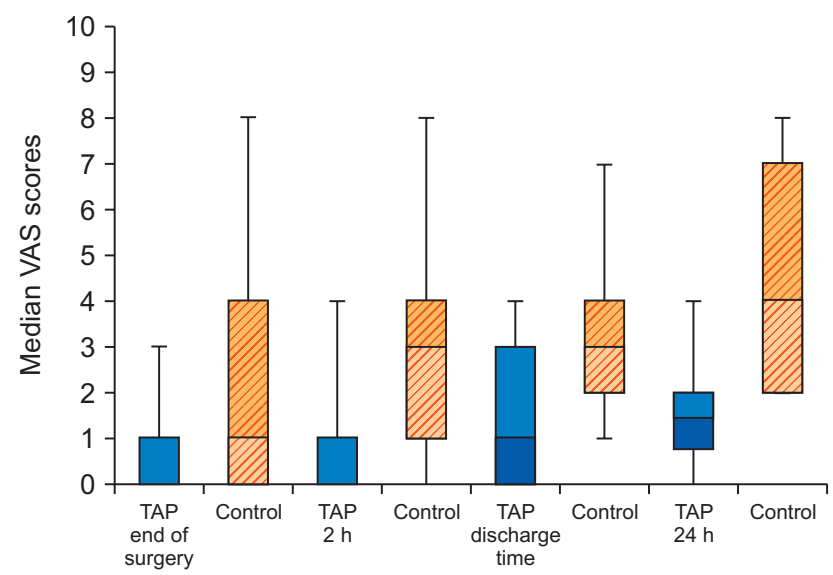

Fig. 1. Median visual analogue scale (VAS) scores at rest (y-axis, 0-10) at different time points during the first 24 hours after surgery ( $\mathrm{x}$-axis). Data expressed as median with the 25th to 75th percentiles (boxes) and the minimum and maximum values (whiskers).

\section{Discussion}

In this study, we demonstrated that the combination of the US-guided TAP and IIN/IHN block is associated with higher intraoperative comfort and better postoperative pain control than the US-guided IIN/IHN block alone when performing Lichtenstein hernioplasty.

The inguinal region is primarily innervated by the IIN and IHN [19]. These two nerves exit the lumbar plexus, leaving the psoas major laterally to travel along the anterior surface of the quadratus lumborum muscle before penetrating the transversus abdominis muscle to travel in the transversalis plane $[19,20]$. In this plane, LA agents exert action in both the IIN/IHN block and the TAP block at different points along the course of the nerves [17]. Mandelkow and Loeweneck [21] described the course of the IIN entering the transversalis plane above the iliac crest midway between the crest and the tip of the 12th rib; the IIN travels with the IHN but consistently enters the transversalis plane $2 \mathrm{~cm}$ anterior to it. However, Jamieson et al. [20] found that the two nerves penetrate the transversus abdominis muscle more anteromedially. The location of the IHN varies with frequent division of the nerves at the level of the iliac crest; therefore, a proximal block of the nerves could theoretically be more effective [21].

IHR induces parietal pain depending on the IHN distribution. Parietal nerve infiltration, usually performed blindly, has been documented to provide pain relief during the first few postoperative hours [22]. However, after the IHN block, the duration of the block is not prolonged enough to allow pain control during the entire postoperative period; pain relief can also be incomplete [22]. Weintraud et al. [23] showed that the landmark-based IIN/IHN block technique results in intramuscular injection in $>80 \%$ of cases with a $40 \%$ failure rate, whereas the US-guided technique is associated with a success rate of $>95 \%$ because of exact intermuscular administration of the LA agent around the nerve structures. Several reports have shown that when using US guidance, greater success can be achieved by more accurate placement of reduced volumes of LA agents closer to the targeted nerves [12,24]. Reduced onset times, longer duration of blocks, and improved postoperative pain scores have also been described for various regional anesthetic techniques using US guidance [24,25].

The TAP is an intermuscular plane located between the internal oblique and transversus abdominis muscles, and the terminal branches of the lower thoracic and first lumbar nerves course through the lateral abdominal wall within this plane [1418]. Injection of LA agents in the TAP may provide unilateral analgesia of the anterior abdominal wall (skin, muscles, and parietal peritoneum) from the T7 to L1 dermatomes [14-18]. The TAP block was first described as a landmark-guided technique, 
and US guidance was subsequently introduced to successfully guide needle insertion and prevent complications related to inadvertent visceral perforation [16-18]. Various studies have demonstrated that the TAP block provides effective analgesia and decreases the postoperative analgesic requirements after caesarian delivery, abdominal hysterectomy, retro-pubic prostatectomy, colorectal surgery, IHR, and abdominal surgery [14-18]. However, further investigations are needed regarding the combination of the TAP block with other regional anesthesia techniques in terms of efficacy and side effects. Aveline et al. [26] compared the efficacy of the US-guided TAP block and conventional "blind" IHN block on both immediate postoperative pain and chronic pain after IHR performed during general anesthesia in ambulatory patients. They showed that the US-guided TAP block provided better immediate postoperative pain relief and reduced the opioid demand but did not reduce the occurrence of chronic pain after hernia repair [26]. Instead, Petersen et al. [27] compared the analgesic effect of the TAP block after primary IHR versus placebo and versus an active comparator (ilioinguinal block and wound infiltration). They failed to demonstrate a reduction in postoperative pain in patients treated with the TAP block [27]. Milone et al. [28] demonstrated the utility of the TAP block to improve the efficacy of conventional "blind" local anesthesia for hernia repair with respect to achieving adequate intraoperative anesthesia, encouraging the use of the US-guided TAP block in hernia surgery. Nevertheless, IIN/IHN blocks have been performed by these authors without US guidance, a technique associated with a high failure rate [23]. Therefore, we can postulate that the better results obtained in the TAP block group may be related to a weak IIN/IHN block caused by the absence of US guidance. In our opinion, US guidance is mandatory to ensure an effective IIN/IHN block.

In the present study, the proportion of patients who achieved adequate anesthesia was higher in the TAP block group. In fact, only $12.5 \%$ of patients in the TAP group required sedation compared with $37.0 \%$ in the IIN/IHN group. The clinical implication of this result is evident: the US-guided TAP block is an effective and feasible anesthesia technique with which to improve local anesthesia and reduce intraoperative discomfort during IHR.

Moreover, postoperative analgesia was more effective in the TAP group at each time point, globally providing superior pain relief at 2 hours, discharge, and 24 hours. Therefore, we found that the US-guided TAP block effectively reduced the postoperative pain and analgesic drug consumption after IHR.

In our opinion, the better intraoperative compliance and lower postoperative pain scores reported in the TAP group could be explained first by the presumable proximal involvement of the IIN/IHN after the injected LA agent had spread above the transverse muscle. Second, muscle relaxation may have been induced by the TAP block, facilitating surgical maneuvers and reducing intraoperative and postoperative pain.

In conclusion, the combination of the US-guided TAP and IIN/IHN block is more effective for intraoperative anesthesia for IHR than is the US-guided IIN/IHN block alone. The addition of the TAP block seems to provide better postoperative analgesia in the 24 hours immediately after surgery. In fact, patients who underwent the TAP block experienced significantly less pain according to their VAS scores. Further investigations are needed to assess the long-term effects of the combination of the US-guided TAP and IIN/IHN block.

Some limitations of the present study need to be outlined. First, a long-term follow-up has not been performed, and data on differences in the prevalence of chronic pain after IHR in the two groups are not available. Second, further trials are needed to address the risks and benefits of TAP block use in the anesthetic and analgesic management of IHR.

\section{ORCID}

Luciano Frassanito, http://orcid.org/0000-0001-6406-2591

Miryam Del Vicario, http://orcid.org/0000-0001-6972-8305

\section{References}

1. Baskerville PA, Jarrett PE. Day case inguinal hernia repair under local anaesthetic. Ann R Coll Surg Engl 1983; 65: 224-5.

2. Callesen T, Bech K, Kehlet H. One-thousand consecutive inguinal hernia repairs under unmonitored local anesthesia. Anesth Analg 2001; 93: 1373-6.

3. White PF, Kehlet H. Improving postoperative pain management: what are the unresolved issues? Anesthesiology 2010; 112: 220-5.

4. Gönüllü NN, Cubukçu A, Alponat A. Comparison of local and general anesthesia in tension-free (Lichtenstein) hernioplasty: a prospective randomized trial. Hernia 2002; 6: 29-32.

5. Nordin P, Zetterström H, Gunnarsson U, Nilsson E. Local, regional, or general anaesthesia in groin hernia repair: multicentre randomised trial. Lancet 2003; 362: 853-8.

6. Perkins FM, Kehlet H. Chronic pain as an outcome of surgery. A review of predictive factors. Anesthesiology 2000; 93: 1123-33.

7. Bay-Nielsen M, Perkins FM, Kehlet H. Pain and functional impairment 1 year after inguinal herniorrhaphy: a nationwide questionnaire study. Ann Surg 2001; 233: 1-7. 
8. Poobalan AS, Bruce J, Smith WC, King PM, Krukowski ZH, Chambers WA. A review of chronic pain after inguinal herniorrhaphy. Clin J Pain 2003; 19: 48-54.

9. Ong CK, Lirk P, Seymour RA, Jenkins BJ. The efficacy of preemptive analgesia for acute postoperative pain management: a meta-analysis. Anesth Analg 2005; 100: 757-73.

10. Bang YS, Park C, Lee SY, Kim M, Lee J, Lee T. Comparison between monitored anesthesia care with remifentanil under ilioinguinal hypogastric nerve block and spinal anesthesia for herniorrhaphy. Korean J Anesthesiol 2013; 64: 414-9.

11. Gofeld M, Christakis M. Sonographically guided ilioinguinal nerve block. J Ultrasound Med 2006; 25: 1571-5.

12. Willschke H, Marhofer P, Bösenberg A, Johnston S, Wanzel O, Cox SG, et al. Ultrasonography for ilioinguinal/iliohypogastric nerve blocks in children. Br J Anaesth 2005; 95: 226-30.

13. Eichenberger U, Greher M, Kirchmair L, Curatolo M, Moriggl B. Ultrasound-guided blocks of the ilioinguinal and iliohypogastric nerve: accuracy of a selective new technique confirmed by anatomical dissection. Br J Anaesth 2006; 97: 238-43.

14. Shin HJ, Kim ST, Yim KH, Lee HS, Sim JH, Shin YD. Preemptive analgesic efficacy of ultrasound-guided transversus abdominis plane block in patients undergoing gynecologic surgery via a transverse lower abdominal skin incision. Korean J Anesthesiol 2011; 61: 413-8.

15. Charlton S, Cyna AM, Middleton P, Griffiths JD. Perioperative transversus abdominis plane (TAP) blocks for analgesia after abdominal surgery. Cochrane Database Syst Rev 2010; (12): CD007705.

16. McDonnell JG, O'Donnell B, Curley G, Heffernan A, Power C, Laffey JG. The analgesic efficacy of transversus abdominis plane block after abdominal surgery: a prospective randomized controlled trial. Anesth Analg 2007; 104: 193-7.

17. McDonnell JG, O'Donnell BD, Farrell T, Gough N, Tuite D, Power C, et al. Transversus abdominis plane block: a cadaveric and radiological evaluation. Reg Anesth Pain Med 2007; 32: 399-404.

18. Ra YS, Kim CH, Lee GY, Han JI. The analgesic effect of the ultrasound-guided transverse abdominis plane block after laparoscopic cholecystectomy. Korean J Anesthesiol 2010; 58: 362-8.

19. van Schoor AN, Boon JM, Bosenberg AT, Abrahams PH, Meiring JH. Anatomical considerations of the pediatric ilioinguinal/ iliohypogastric nerve block. Paediatr Anaesth 2005; 15: 371-7.

20. Jamieson RW, Swigart LL, Anson BJ. Points of parietal perforation of the ilioinguinal and iliohypogastric nerves in relation to optimal sites for local anaesthesia. Q Bull Northwest Univ Med Sch 1952; 26: 22-6.

21. Mandelkow H, Loeweneck $H$. The iliohypogastric and ilioinguinal nerves. Distribution in the abdominal wall, danger areas in surgical incisions in the inguinal and pubic regions and reflected visceral pain in their dermatomes. Surg Radiol Anat 1988; 10: 145-9.

22. Møiniche S, Mikkelsen S, Wetterslev J, Dahl JB. A qualitative systematic review of incisional local anaesthesia for postoperative pain relief after abdominal operations. Br J Anaesth 1998; 81:377-83.

23. Weintraud M, Marhofer P, Bösenberg A, Kapral S, Willschke H, Felfernig M, et al. Ilioinguinal/iliohypogastric blocks in children: where do we administer the local anesthetic without direct visualization? Anesth Analg 2008; 106: 89-93.

24. Willschke H, Bösenberg A, Marhofer P, Johnston S, Kettner S, Eichenberger U, et al. Ultrasonographic-guided ilioinguinal/iliohypogastric nerve block in pediatric anesthesia: what is the optimal volume? Anesth Analg 2006; 102: 1680-4.

25. Marhofer P, Greher M, Kapral S. Ultrasound guidance in regional anaesthesia. Br J Anaesth 2005; 94: 7-17.

26. Aveline C, Le Hetet H, Le Roux A, Vautier P, Cognet F, Vinet E, et al. Comparison between ultrasound-guided transversus abdominis plane and conventional ilioinguinal/iliohypogastric nerve blocks for day-case open inguinal hernia repair. Br J Anaesth 2011; 106: 380-6.

27. Petersen PL, Mathiesen O, Stjernholm P, Kristiansen VB, Torup H, Hansen EG, et al. The effect of transversus abdominis plane block or local anaesthetic infiltration in inguinal hernia repair: a randomised clinical trial. Eur J Anaesthesiol 2013; 30: 415-21.

28. Milone M, Di Minno MN, Musella M, Maietta P, Salvatore G, Iacovazzo C, et al. Outpatient inguinal hernia repair under local anaesthesia: feasibility and efficacy of ultrasound-guided transversus abdominis plane block. Hernia 2013; 17: 749-55. 